\title{
Evaluación de la percepción del riesgo ocupacional en trabajadores de una empresa del sector de la construcción en Bogotá D.C.
}

\author{
Evaluation of risk perception occupational in workers of a company \\ of construction in Bogotá D.C.
}

Yuri Lilian González ${ }^{1}$.

\section{Resumen}

Objetivo. Evaluar la percepción del riesgo en trabajadores de una empresa del sector de la construcción en la ciudad de Bogotá D.C. desde el paradigma psicométrico. Materiales y métodos. Se aplicaron dos cuestionarios, los cuales permitieron identificar las actividades que los trabajadores consideraban como más peligrosas, su magnitud de riesgo y los atributos que subyacen a la percepción. Resultados. Los hallazgos indican que los trabajadores pueden estar considerando con mayor frecuencia el atributo de inmediatez de las consecuencias para determinar la gravedad del dańo, que al grado de temor o rechazo por aquellas actividades que se consideren como más dañinas para la salud y el bienestar, de igual forma se evidenció que se percibe una magnitud del riesgo alta preferiblemente en aquellas actividades novedosas para los trabajadores y se encontró que aunque la magnitud del riesgo es alta, perciben control sobre los riesgos y un conocimiento de los mismos que les permite enfrentarse a determinados peligros sin temor a causar daños en su salud.

Palabras clave: Paradigma Psicométrico, percepción del riesgo, riesgo, trabajadores de la construcción.

\begin{abstract}
Objective. Assessing risk perception among employees of a company in the construction sector in Bogota DC from the psychometric paradigmMaterials and methods. Two questionnaires were applied, which helped identify the activities that workers considered as more dangerous, the magnitude of risk and the attributes that underlie perception. Results. The findings indicate that workers may be considering more often attributes of immediacy of consequences to determine the severity of the damage that the degree of fear or rejection by those activities which are considered as more harmful to health and wellness. In the same way became clear that a magnitude of high risk is perceived preferably in newfangled activities for workers and found that although the magnitude of the risk is high, perceived control over risks and knowledge of them that allows them to take risk without fear of causing damage to your health
\end{abstract}

Keywords: psychometric paradigm, Risk perception, risk, constructions workers.

1Psicóloga - Magíster en Salud y Seguridad en el trabajo, Docente Universidad Nacional Abierta y A Distancia - UNAD. ORCID. 0000-0001-5625-7508

Correspondencia: yuri.gonzalez@unad.edu.co

Recibido: 07/04/2015 Aceptado: 05/06/2015 


\section{Introducción}

La percepción del riesgo como proceso cognitivo y social en los seres humanos puede afectar la forma como las personas interactúan con los diversos factores de riesgo en su actividad laboral, considerándolos como poco dañinos para su salud, además de sentir tener el control y el conocimiento sobre ellos, lo que podría llevar a desencadenar un accidente laboral.

En este contexto Morillejo (1) plantea que la percepción del riesgo en el ámbito laboral se convierte en un elemento crucial para entender las prácticas de trabajo inseguras de los trabajadores, donde intervienen no solo las variables cognitivas sino también las psicosociales. Lo que indica que este proceso está bajo la influencia de los conocimientos previos, los patrones culturales y factores del medio en el que las personas se desenvuelven (2).

Al respecto Morillejo (1) y Sjöberg (3) exponen que la conducta preventiva de las personas está en función de la amenaza percibida y de los beneficios que pueda recibir por llevarla a cabo. A su vez esta percepción depende de la susceptibilidad y de la gravedad de las consecuencias que los sujetos perciben al experimentar un accidente laboral. Por consiguiente un trabajador tomará medidas de prevención dependiendo de lo vulnerable o no que se perciba.

Dentro del estudio de la percepción del riesgo se destacan cuatro enfoques: Un enfoque centrado en aspectos psicológicos, un segundo enfoque centrado en aspectos psicosociales, un tercer enfoque centrado en aspectos culturales y el cuarto enfoque denominado paradigma psicométrico (4).

Enfoque centrado en aspectos psicológicos: Bajo este enfoque surgen los primeros estudios de la percepción del riesgo, centrados básicamente en conceptos de la psicología cognitiva. Entiende la percepción como sesgos cognitivos de las personas a la hora de tomar una decisión. Por otro lado también surgen los estudios desde la teoría de la personalidad, enfocados al estudio de las características de personalidad, que según sus exponentes hace que los sujetos asuman o eviten los riesgos (5).

Los estudios realizados desde este enfoque se centran en el individuo y conciben el concepto de percepción del riesgo como la evaluación que hace un sujeto sobre la probabilidad de que ocurra un evento adverso y sus consecuencias, sin tener en cuenta otros procesos que subyacen al ser humano como sus creencias y emociones.

En este enfoque se destaca el establecimiento de una taxonomía de los riesgos y la identificación de las características que se relacionan con la estimación de la magnitud de los mismos(6).

Enfoque centrado en aspectos psicosociales: Bajo este enfoque la percepción del riesgo es vista como una actitud que asumen las personas frente a los peligros que se enfrenta de acuerdo a sus valores y creencias (4). Desde este punto de vista las investigaciones se han centrado en desarrollar escalas para medir la actitud de las personas frente a los peligros. Los estudios más clásicos se han realizado en el tema de la energía nuclear y la industria petroquímica.

En este enfoque también son introducidas las características socio demográficas de los individuos con el objetivo de buscar relaciones entre estas y la percepción del riesgo, Bickerstaff (7) plantea que estos estudios se centran en la realización de encuestas sobre actitud y en sus resultados se encontró que las personas reconocen la presencia del peligro pero no el daño que les pueda causar. 
Slovic (8) concluye que si se conoce el riesgo percibido por las personas, se podrían anticipar muchos de los comportamientos riesgosos que son adquiridos y determinar el conocimiento que tienen las personas sobre el riesgo al que se enfrentan. Esto sería fundamental para poder intervenir en ellos.

Enfoque centrado en aspectos sociológicos: Desde este enfoque la percepción del riesgo es entendida como el estudio de las creencias, actitudes, juicios, sentimientos, valores, disposiciones sociales y culturales que tienen las personas frente a los factores de riesgos y los beneficios que trae exponerse a ellos (5).

Esta concepción plantea que percibir el riesgo no es un proceso meramente matemático que depende del número de consecuencias adversas ocurridas, sino que es un proceso multidimensional en los que las personas evalúan diversas características como son: potencial catastrófico, carácter voluntario o no de la exposición, grado de confianza o credibilidad que tienen las personas que intervienen en la gestión entre otras, lo que contribuye a valorar finalmente la aceptación o no la exposición a los factores de riesgo.

Enfoque psicométrico de la percepción del riesgo: Se desarrolla a mediados de los ańos 70 y surge a partir de los estudios realizados desde los enfoques anteriores. Por un lado introduce en sus investigaciones algunas características sociodemográficas y actitudinales de los sujetos de estudio y por el otro lado introduce las características cualitativas referidas a las dimensiones sociales para evaluar los riesgos. Por lo tanto se podría decir que este enfoque recoge aspectos de los enfoques psicosociales y sociológicos. Uno de sus planteamientos es que el riesgo percibido se puede estudiar por medio de técnicas de escalamiento psicométrico, de allí que lleve su nombre de paradigma psicométrico (8).
Los estudios realizados desde este enfoque se hacen a través de la utilización de cuestionarios que permiten a los sujetos juzgar de un listado de actividades, la magnitud del riesgo percibido y los atributos psicosociales del mismo (por ejemplo, posibilidad de control, gravedad de las consecuencias, temor, etc.).

A partir de las respuestas emitidas por los sujetos los análisis se centran en comprender la relación entre los diferentes atributos y su poder explicativo sobre la estimación de la magnitud del riesgo percibido.

Desde el enfoque psicométrico los atributos psicosociales se refieren a una serie de características de los riesgos, las cuales son valoradas por las personas en el momento asumir el riesgo (9).

Las investigaciones iniciales desde el paradigma psicométrico incluyeron un total de nueve atributos (4). Algunos estudios posteriores incluyeron hasta diez y ocho atributos.

Las investigaciones sobre percepción del riesgo han encontrado que los riesgos que son fácilmente aceptados están asociados a actividades vistas como altamente beneficiosas para la personas y no necesariamente esta percepción se relaciona con el estado de salud de las personas(10). En este sentido se podría considerar que muchas de las condiciones inseguras a nivel laboral pueden ser toleradas por el trabajador y vistas como parte inevitable del mismo, partiendo de los beneficios encontrados de su actividad, como la remuneración económica, el estatus social, etc.(8).

La misma situación es planteada por Blanco y colaboradores(11), cuando expone que en la toma de decisiones para realizar una conducta, la persona tenderá a aquella que le genere mayor utilidad. Así se podría llegar a plantear que un trabajador se enfrentará a una situación riesgosa 
más fácilmente si de la misma, obtendrá algún beneficio, por ejemplo mayor remuneración, ahorro de tiempo, aceptabilidad social, etc.

Elkind(12) en un estudio de percepción del riesgo en el sector agrícola encontró que los trabajadores reconocen que su labor proporciona alta probabilidad de riesgos, sin embargo, consideran que gracias a la realización de esta actividad ellos obtienen calidad de vida para sí mismos y su familia. Así, los beneficios alcanzados se logran a través de la aceptación de los riesgos, minimizando los mismos, frente a los beneficios recibidos por la realización de la labor. Este autor concluye en su investigación que la percepción del riesgo es el resultado de un análisis costo beneficio que se basa en el conocimiento subjetivo de las personas el cual está influenciado por condiciones políticas, económicas y culturales.

Bickerstaff (7)considera que adicionalmente a lo planteado por los estudios de carácter psicológico y psicosocial se sugiere reconocer la importancia de incluir variables de tipo cultural y social con el fin de ampliar los enfoques anteriores. De esta forma, el estudio de la percepción del riesgo ya no solo se trata como un proceso cognitivo o de actitudes sino que también es un proceso social y cultural, que se realiza con el fin de contextualizar el estudio de la percepción del riesgo. En este sentido Puy (4)argumenta que los criterios utilizados por las personas para valorar la magnitud del riesgo atienden a dimensiones psicológicas, sociales y culturales que trascienden a solo considerar la probabilidad de causar daño o muerte.

Arezes y colaboradores(13), encontraron que los trabajadores no consideraban los altos niveles de ruido como peligroso para su salud y por lo tanto no era necesario el uso de protectores auditivos durante la realización de su labor, con lo cual sustenta que la percepción del riesgo debe ser considerada como un tema central en el diseño e implementación de los programas para la conservación de la salud, indicando que entender la manera en que los trabajadores perciben el riesgo es fundamental para una mejor gestión del riesgo.

El estudio de la percepción del riesgo se constituye en un desarrollo fundamental para la formulación y planeación de actividades que contribuyan a la prevención de los efectos adversos en la salud, conocer la percepción de los trabajadores sobre los riesgos a los que diariamente están sometidos, permite obtener un conocimiento más real y amplio para ser utilizado en la formulación de estrategias de intervención en salud y seguridad en el trabajo enfocadas al punto de vista de los trabajadores implicados en el proceso(14).

\section{Materiales y métodos}

Se realizó un estudio descriptivo de abordaje cuantitativo orientado a la evaluación de la percepción del riesgo mediante la identificación de los atributos psicosociales en los trabajadores de una empresa del sector de la construcción de edificaciones para uso residencial.

El estudio se realizó con trabajadores que realizaban las diferentes actividades laborales del proceso de trabajo de la construcción como: Preparación del terreno, cimentación, levantamiento de estructuras, mampostería, colocación de techos, instalaciones hidráulicas, instalaciones eléctricas y acabados; en una empresa de la ciudad de Bogotá D.C. Para la escogencia de la muestra solo se tuvo en cuenta los trabajadores con cargos de: maestro, oficial y ayudante de obra.

El proceso de selección de la muestra guardó concordancia con el de recolección de la información 
en dos momentos, tal y se describe a continuación:

La identificación de las actividades peligrosas percibidas por los trabajadores.

La estimación cuantitativa de la magnitud del riesgo percibido y la valoración de los atributos del riesgo percibidos.

Para cada una de estas etapas se seleccionó una muestra representativa de carácter aleatorio con base en los criterios de muestreo para poblaciones finitas, de acuerdo a los fines que se buscaba en cada una de ellas.

Para la primera etapa se seleccionó una muestra de 385 trabajadores distribuidos en 12 proyectos de vivienda a partir de los siguientes parámetros: Intervalo de confianza del 95\% y error máximo admisible de 0.05 .

De los 385 sujetos seleccionados el 94\% (360 hombres) correspondía al género masculino y el 6\% al género femenino (25 mujeres). Es decir el mayor porcentaje correspondió al género masculino.

La edad de los participantes del estudio correspondió en su mayor porcentaje a trabajadores en edad entre los 26 y los 35 ańos con un total del 35\%. La edad de los trabajadores osciló entre los 18 y los 72 años, con una media de 35 años y una deviación estándar de 10,8.

En la segunda etapa del estudio la muestra seleccionada correspondió a 213 trabajadores distribuidos en 9 proyectos de soluciones de vivienda. El procedimiento para la selección del número de la muestra se determinó a partir de los datos generados por una muestra piloto, la cual a partir de los datos recogidos permitió establecer una muestra final con los siguientes parámetros estadísticos:
Intervalo de confianza: $95 \%$, error máximo admisible: 0.2 y mayor varianza obtenida en la muestra piloto: 1,8

De los 213 sujetos seleccionados el 98\% (209 hombres) correspondía al género masculino y el 2\% (4 mujeres) correspondía al género femenino.

La edad de los participantes del estudio correspondió en su mayor porcentaje a trabajadores en edad entre los 26 y los 35 años con un total del $39 \%$. La edad de los trabajadores osciló entre los 18 y los 64 ańos, con una media de 34 años y una deviación estándar de 10,2.

Las técnicas que se siguieron en el estudio se basan en el modelo denominado Paradigma Psicométrico (8), el cual permitió obtener:

El listado de actividades peligrosas percibidas.

La estimación cuantitativa de la magnitud del riesgo percibido.

La valoración de los atributos del riesgo percibido.

\section{Instrumentos}

Actividades peligrosas percibidas: Para esta categoría se desarrolló un juicio de expertos y una revisión de la literatura relacionada con este ítem, que permitió identificar un listado de actividades consideradas como peligrosas en empresas dedicadas a la construcción de edificaciones para uso residencial.

Una vez identificadas estas actividades, se presentó a cada trabajador participante del estudio el listado de las mismas para que a su juicio señalara las que él se consideraba directamente expuesto. Algunos trabajadores que manifestaron no saber leer, ni escribir, recibieron la ayuda del investigador para su diligenciamiento. 
Estimación cuantitativa de la magnitud del riesgo percibido y valoración de los atributos del riesgo percibido: Para la identificación de estos aspectos se utilizó un cuestionario adaptado de la investigación realizada por Puy (4) en su estudio sobre percepción social del riesgo.

Una vez identificadas las actividades peligrosas a las que se consideraron expuestos los trabajadores participantes del estudio, se escogieron las diez actividades mencionadas por más del 50\% de los trabajadores y se procedió a la adaptación del instrumento donde finalmente se evaluaron solo las actividades seleccionadas.

En esta fase, la tarea de los sujetos consistía en valorar la magnitud del riesgo percibido con criterios adoptados a partir de la metodología del paradigma psicométrico que establece una escala de graduación donde 0 corresponde a "sin riesgo" y 100 corresponde a "riesgo extremo". Para este estudio se adoptó una escala de 0 a 5 , donde 0 correspondió a que no se presenta daño en la salud del trabajador y 5 puede ocurrir la muerte del trabajador.

Una vez estimada la magnitud del riesgo percibido, los sujetos de estudio debían valorar siete de los atributos establecidos por el paradigma psicométrico en el estudio de la percepción del riesgo. Estos atributos hacen referencia a: Inmediatez del efecto, conocimiento del riesgo por los expuestos, conocimiento del riesgo por parte del encargado de Salud y Seguridad de la empresa, posibilidad de control, familiaridad / novedad, potencial catastrófico y temor. Para la valoración de estos atributos se utilizó una escala tipo Likert.

Esta fase de recolección de información se realizó de manera personalizada con el trabajador, en forma de entrevista. La duración de cada entrevista fue de aproximadamente 40 minutos.

\section{Resultados \\ Identificación de las actividades peligrosas}

Se identificaron ciento setenta y nueve (179) actividades peligrosas realizadas en el proceso de construcción de edificaciones para uso residencial. Una vez identificadas estas actividades se realizó un proceso de depuración donde se seleccionaron actividades que podían ser contenidas en otras y actividades que representaban riegos similares. Con este procedimiento se depuró hasta tener una lista de treinta y una actividades peligrosas la cual fue la seleccionada para presentar a la primera muestra de trabajadores.

De las treinta y una actividades presentadas a la primera muestra de trabajadores se seleccionaron finalmente las diez (10) actividades que iban a formar parte de la segunda etapa del estudio. Estas actividades finales fueron escogidas de acuerdo a los resultados de frecuencia obtenidos con las respuestas de los trabajadores.

Como se puede observar en la Tabla 1 para los trabajadores participantes en el estudio, las actividades peligrosas en el proceso de la construcción corresponden en primer lugar al trabajo en Alturas con un porcentaje de $78 \%$ (300 trabajadores) y en segundo lugar al trabajo en ambientes de mucho ruido con el 68\% (260 trabajadores). Resultados similares a los encontrados en el estudio realizado por el seguro social para Colombia(15), donde el mayor porcentaje de los trabajadores elige el trabajo en alturas como el mayor peligro en la construcción. 
Tabla 1. Listado de actividades peligrosas percibidas por los trabajadores

\section{ACTIVIDADES PELIGROSAS} No TRABAJADORES
FRECUENCIA /

PORCENTAJE $\%$

$78 \%$

1. Trabajar en alturas 300 260 $68 \%$

2. Trabajar en ambientes ruidosos 255

3. Trabajos con exposición a polvo

4. Uso de herramientas cortantes 254 $66 \%$

5. No usar los elementos de protección personal 224 $66 \%$

6. Trabajar en espacios de baja iluminación 221 $58 \%$

7. Uso de instalaciones eléctricas en mal estado.

8. Trabajo con exposición a gases y vapores 216 201 $57 \%$

9. Mantener desorden en la obra 200

10. Traslado o manipulación de herramientas u objetos pesados

199

199

190

185

$56 \%$

11. Trabajar en Posturas forzadas

12. Mantener desaseo en la obra 181

180

$52 \%$

13. Uso de instalaciones eléctricas sin protección.

14. Ausencia de señalización

15. Levantamiento de cargas

16. Trabajar con Posturas incomodas 177

17. Trabajar en excavaciones 176 $52 \%$

18. Uso de instalaciones eléctricas provisionales. 174 $52 \%$ $49 \%$

19. Utilización de cemento

20. Utilización de explosivos

21. Uso de herramientas incomodas

22. Trabajar en altas temperaturas 174 167 167 $48 \%$ $47 \%$ $47 \%$ $46 \%$

23. Uso de equipos que producen vibraciones

24. Manejo de soldadura

25. Exposición a radiaciones 150 148 $46 \%$ $45 \%$ $45 \%$ $43 \%$

26. Uso de herramientas que producen vibraciones 147 146 $43 \%$ $39 \%$ $38 \%$ $38 \%$

27. Uso de herramientas rudimentarias

28. Uso de herramientas improvisadas

29. Movimientos repetitivos 143 $38 \%$ $37 \%$ 136 $35 \%$ 135 $35 \%$ 133 $35 \%$

30. Utilización de pinturas 107 $28 \%$ 31. Utilización de asfalto 31. Utilización de asfalto 105 $27 \%$

Fuente: autor 
Para la segunda etapa de la investigación se escogieron las actividades señaladas por más del $50 \%$ de los trabajadores, lo que generó un listado de diez actividades peligrosas con las cuales finalmente se procedió a realizar la identificación de los atributo psicosociales.
La Tabla 2 presenta el listado definitivo de actividades que fueron seleccionadas para la realización de la segunda etapa del estudio. Estimación cuantitativa de la magnitud del riesgo y valoración de los atributos del riesgo percibido

Tabla 2. Listado definitivo de las actividades seleccionadas para la segunda etapa del estudio

\section{ACTIVIDADES PELIGROSAS}

1. Trabajar en alturas (ACT 1$)$

300

$78 \%$

2. Trabajar en ambientes ruidosos (ACT 2)

260

$68 \%$

3. Trabajos con exposición a polvo (ACT 3)

255

$66 \%$

4. Uso de herramientas cortantes (ACT 4)

254

$66 \%$

5. No usar los elementos de protección personal (ACT 5)

224

$58 \%$

6. Trabajar en espacios de baja iluminación (ACT 6)

221

$57 \%$

7. Uso de instalaciones eléctricas en mal estado. (ACT 7)

216

$56 \%$

8. Trabajo con exposición a gases y vapores (ACT 8)

201

$52 \%$

9. Mantener desorden en la obra (ACT 9)

200

$52 \%$

10. Traslado o manipulación de herramientas u objetos pesados (ACT 10)

199

$52 \%$

Fuente: autor

Los resultados del instrumento aplicado a los trabajadores permitieron la realización de un perfil comparativo de la magnitud del riesgo percibido y sus atributos para cada una de las actividades que se revisaron.

\section{Magnitud del riesgo percibido:}

Con relación a este ítem evaluado, los resultados muestran en una escala de 0 a 5 (donde 0 corresponde a que no se presenta daño en la salud del trabajador y 5 puede ocurrir la muerte del trabajador), una media por encima de 3,0 para todas las actividades evaluadas. La actividad trabajar en alturas (ACT 1) fue la que obtuvo el mayor resultado entre todas las actividades con una media de 5,0.

Las actividades de trabajar en ambientes ruidosos (ACT 2) y trabajos con exposición a polvo (ACT 3) fueron las que menor resultado obtuvieron en este ítem con una media de 3,3 para cada una.

La Gráfica 1 presenta los resultados obtenidos ara todas las actividades evaluadas. 
Gráfica 1. Resultados de la magnitud del riesgo percibido para cada uno de las actividades

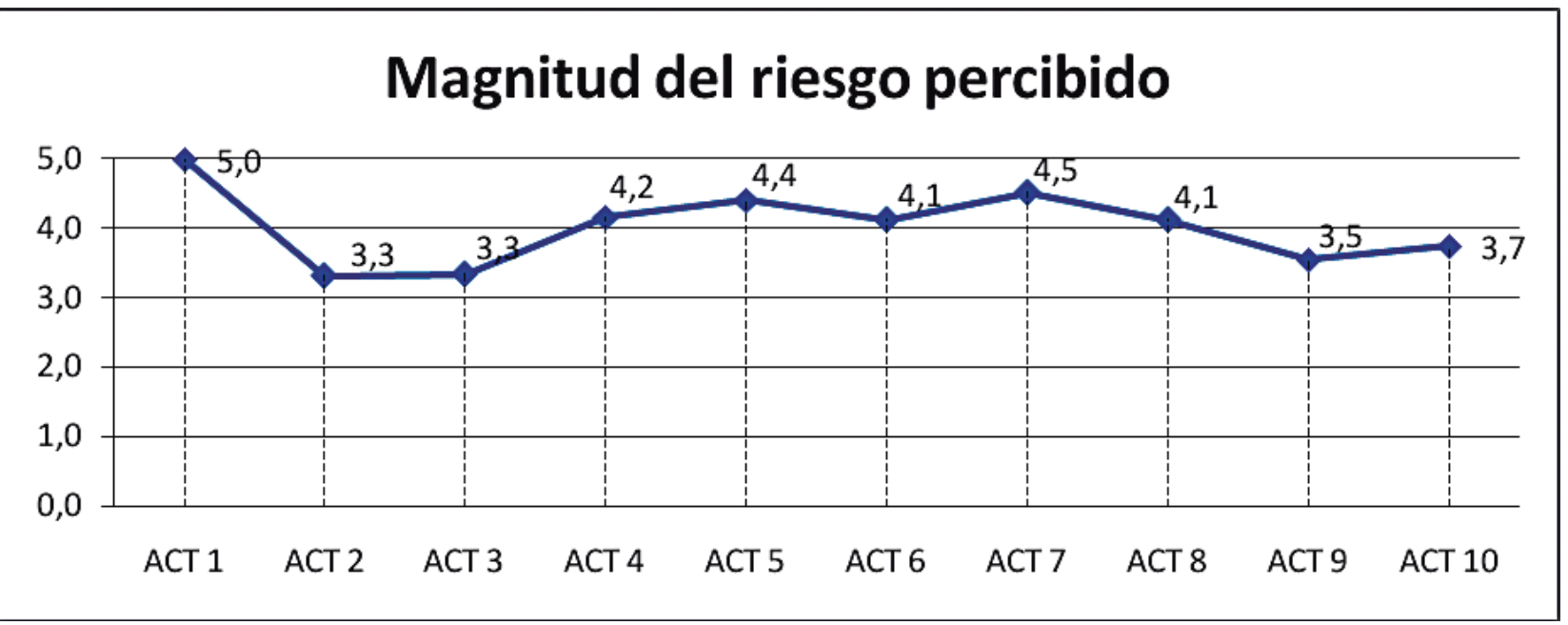

Fuente: autor

La Tabla 3 presenta la media y las desviaciones estándar para cada una de las actividades con relación a la magnitud del riesgo percibido.

Tabla 3. Resultados de la Magnitud del Riesgo Percibido por los trabajadores

\begin{tabular}{|c|c|c|}
\hline ACTIVIDADES PELIGROSAS & MEDIA & $\begin{array}{l}\text { DESVIACIÓN } \\
\text { ESTANDAR }\end{array}$ \\
\hline 1. Trabajar en alturas & 5,0 & 0,2 \\
\hline 2. Trabajar en ambientes ruidosos & 3,3 & 0,9 \\
\hline 3. Exposición a polvo & 3,3 & 1,2 \\
\hline 4. Uso de herramientas cortantes & 4,2 & 1,2 \\
\hline 5. No usar los elementos de protección personal & 4,4 & 1,0 \\
\hline 6. Trabajar en espacios de baja iluminación & 4,1 & 1,0 \\
\hline 7. Uso de instalaciones eléctricas en mal estado & 4,5 & 0,9 \\
\hline 8. Exposición a gases y vapores & 4,1 & 1,0 \\
\hline 9. Desorden en la obra & 3,5 & 1,2 \\
\hline 10. Traslado o manipulación de herramientas u objetos pesados & 3,7 & 0,9 \\
\hline
\end{tabular}

Fuente: autor 
Como se puede apreciar en la tabla anterior las desviaciones estándar más pequeñas, por debajo de 1,0 corresponden a las actividades de trabajar en alturas, trabajar en ambientes ruidosos, usar instalaciones eléctricas en mal estado y trasladar y manipular herramientas u objetos pesados. Es decir en estas actividades los resultados se presentan de manera más homogénea con relación a la magnitud del riesgo percibido por los trabajadores.

\section{Valoración de los atributos del riesgo percibido:}

Inmediatez del efecto

Los trabajadores consideran como las actividades que presentan un efecto en la salud de manera más inmediata las siguientes: El uso de herramientas cortantes $(X=4,4$ y $S=1,0)$, seguido por trabajar en alturas $(\mathrm{X}=4,3$ y $S=1,0)$ y no usar elementos de protección personal $(\mathrm{X}=4,3$ y $\mathrm{S}=1,2)$. Las actividades que presentan efectos más demorados a la salud corresponden a exponerse a polvo $(\mathrm{X}=3,0$ y $S=1,6)$ y trabajar en ambientes ruidosos $(\mathrm{X}=2,7$ y $\mathrm{S}=1,5)$.

Conocido por los expuestos

En general los trabajadores manifiestan que las diferentes actividades que representan un riesgo para su salud son conocidas por ellos. La media mínima obtenida en este ítem corresponde a la exposición a gases y vapores con 4,4 .

Conocido por parte del encargado de Salud y Seguridad en la Empresa

Los trabajadores perciben que los riesgos generados por las actividades evaluadas también son conocidos por los expertos en el tema, en este caso el responsable de salud y seguridad en cada uno de los proyectos evaluados. Como se puede observar en la Gráfica 2 el valor de la media oscila entre 4,8 y 4,9.

Posibilidad de control

Con relación al atributo de posibilidad de control, los resultados indican que los trabajadores consideran que poseen el control y tienen la posibilidad de prevenir los riesgos generados por dichas actividades. En este atributo la media de menor valor corresponde al no uso de elementos de protección individual con 4,1 y una desviación estándar de 1,6.

\section{Familiaridad / novedad}

En el atributo de familiaridad los resultados indican que para los trabajadores las actividades más antiguas y familiares corresponden a trabajar en ambientes ruidosos $(X=4,3$ y $S=1,2)$ y exposición a polvo $(\mathrm{X}=4,3$ y $\mathrm{S}=1,3)$. La actividad que fue considerada las más novedosa para los trabajadores y menos familiar correspondió al uso de instalaciones eléctricas en mal estado $(\mathrm{X}=1,7$ y $\mathrm{S}=1,4)$.

\section{Potencial catastrófico}

Con relación al atributo de potencial catastrófico se encontró que las actividades que afectan a un gran número de trabajadores al mismo tiempo, según los sujetos de estudio corresponden a trabajar en espacios con baja iluminación ( $\mathrm{X}=4,0$ y $\mathrm{S}=1,3)$, el uso de instalaciones eléctricas en mal estado $(X=4,0$ y $S=1,3)$ y la exposición a gases y vapores $(\mathrm{X}=4,0$ y $\mathrm{S}=1,2)$.

Los resultados también indican que la actividad con menor potencial catastrófico corresponde al uso de herramientas cortantes $(\mathrm{X}=3,1$ y $S=1,4)$.

\section{Temor}

De acuerdo a las respuestas emitidas por los trabajadores, la actividad por la que sienten mayor temor por los daños a la salud que pueden obtener es el no uso de los elementos de protección individual $(\mathrm{X}=4,7$ y $S=0,7)$.

Las actividades por la que la preocupación es menor corresponden a trabajo en alturas $(\mathrm{X}=3,8$ y $\mathrm{S}=1,6)$ y uso de herramientas cortantes $(\mathrm{X}=3,8$ y $\mathrm{S}=1,6)$.

La Tabla 4 presenta la media y las desviaciones estándar de los resultados obtenidos para cada uno de los atributos evaluados. 
Tabla 1. Listado de actividades peligrosas percibidas por los trabajadores

\begin{tabular}{|cccccccccccccccc} 
& \multicolumn{2}{c}{$\begin{array}{c}\text { Inmediatez } \\
\text { del Efecto }\end{array}$} & \multicolumn{2}{c}{$\begin{array}{c}\text { Conocido por } \\
\text { Expuestos }\end{array}$} & \multicolumn{2}{c}{$\begin{array}{c}\text { Conocido } \\
\text { por SST }\end{array}$} & \multicolumn{2}{c}{$\begin{array}{c}\text { Posibilidad } \\
\text { de Control }\end{array}$} & \multicolumn{2}{c}{ Familiar } & \multicolumn{3}{c}{$\begin{array}{c}\text { Potencial } \\
\text { Catastrófico }\end{array}$} & \multicolumn{2}{c}{ Temor } \\
\hline
\end{tabular}

Fuente: autor

La Gráfica 3 presenta los resultados obtenidos para todas las actividades en relación a la estimación cuantitativa del riesgo y la valoración de los atributos. En esta gráfica se observa que para los atributos de conocimiento por los expuestos, conocimiento por los expertos y posibilidad de control, las medias obtenidas por cada actividad son similares, mientras que para los atributos de inmediatez del efecto, familiaridad, potencial catastrófico y temor las medias obtenidas varían en cada actividad evaluada.

Gráfica 1. Resultados de la magnitud del riesgo percibido para cada uno de las actividades

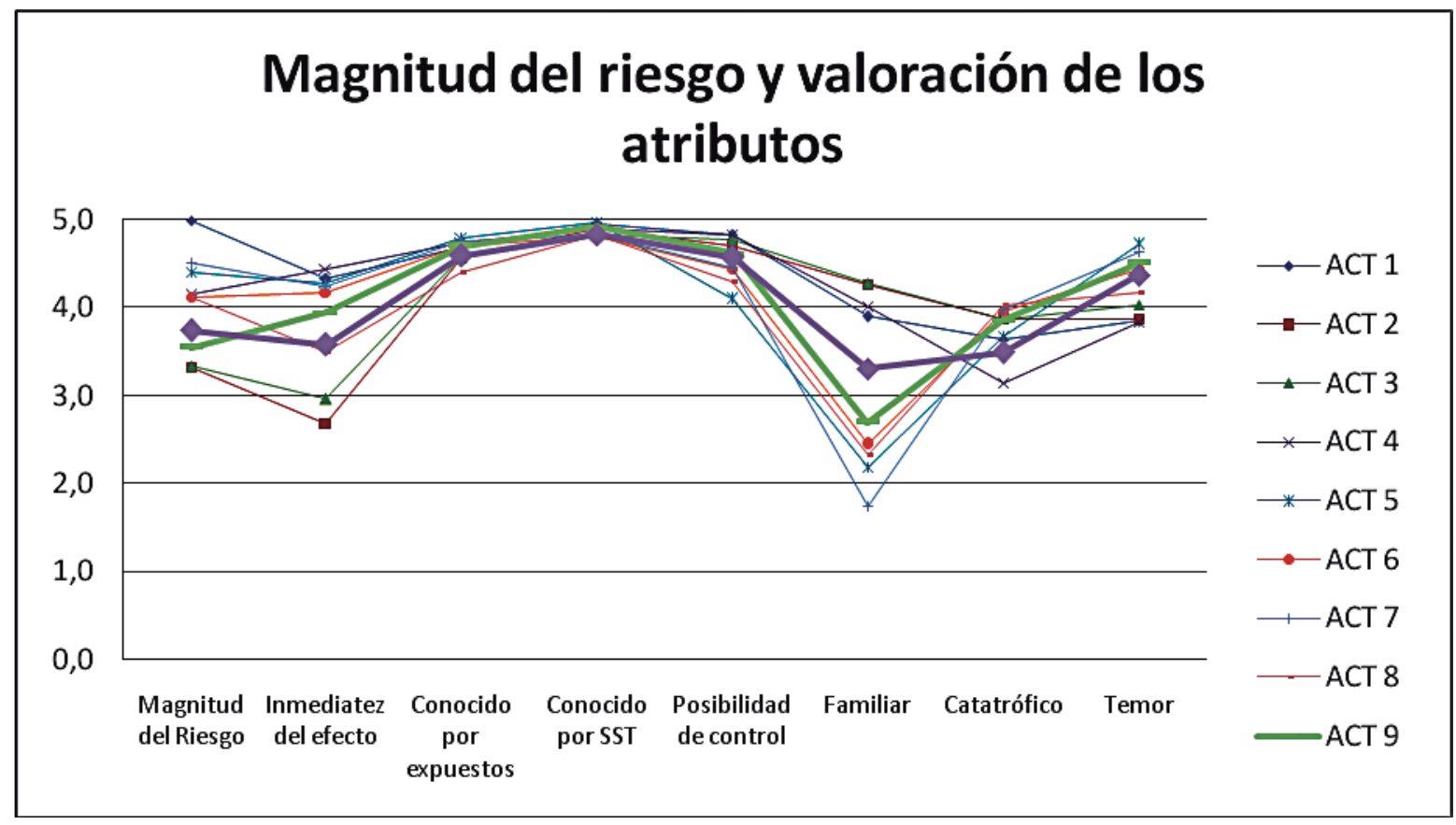


Los resultados expuestos indican la existencia de actividades percibidas por los trabajadores con una mayor magnitud del riesgo. El trabajo en alturas ocupa el primer lugar tanto en el listado de las actividades peligrosas percibidas, como en la estimación cuantitativa del riesgo. Con relación a los atributos el trabajo en alturas se percibe también como una actividad con una inmediatez de efecto alta.

Las actividades de exponerse a ruido y exponerse a polvo, son aquellas percibidas con una magnitud del riego baja, es decir los trabajadores perciben que los daños a su salud no son tan graves, pero también manifiestan que sus efectos a la salud son demorados, lo contrario al trabajo en alturas. Con relación al atributo familiaridad, estas actividades son las que los sujetos manifiestan como más antiguas para ellos y menos novedosas.

En general aquellas actividades vistas con una magnitud de riesgo alto fueron también percibidas por los trabajadores con una inmediatez del efecto alto, a excepción de la exposición de gases y vapores que se percibe con un una gravedad de sus afectos alta pero con una inmediatez un poco más baja.

Con relación al no uso de los elementos de protección personal, esta actividad fue la más temida por los trabajadores y también fue considerada como la actividad con menor posibilidad de control. Cabe anotar aquí que el uso de estos elementos generalmente depende del trabajador, es él quien decide si usarlos o no, pero cuando se percibe que su posibilidad de control es baja y que en algunas ocasiones no depende de ello, podría aludir al hecho de que no son entregados sus elementos para hacer uso de ellos o que no hay una frecuencia establecida para su reposición y adecuado mantenimiento de los mismos.

Si se revisan los resultados de la magnitud del riesgo percibido y el atributo de familiaridad, se encuentra que en las actividades evaluadas, cuando la magnitud del riesgo es percibida como alta, son actividades novedosas para los trabajadores y en actividades antiguas y familiares para los trabajadores la magnitud del riesgo es percibida como baja. Por ejemplo el caso de la exposición a polvo y la exposición a ruido mencionados anteriormente o el uso de instalaciones eléctricas donde se considera una actividad novedosa con una magnitud del riesgo alta.

Los atributos de conocido por los expuestos, conocido por los expertos y posibilidad de control, en general obtuvieron resultados que indican que los trabajadores perciben que existe un conocimiento por parte de ellos y por parte de los expertos que es suficiente para su prevención y adicionalmente, una posibilidad de control que les permite tomar acciones que prevengan el daño a la salud causado por estas actividades.

De acuerdo a los estudios realizados por Slovic y colaboradores(16), se encontró que uno de los atributos que más se relacionaba con la magnitud del riesgo percibido correspondía al temor, lo que se evidencia en que las actividades más temidas por los sujetos de estudio eran también las que percibían con una magnitud de riesgo mayor.

Los hallazgos de este estudio indican que solamente para las actividades de no usar los elementos de protección individual, el trabajar en espacios de baja iluminación, el uso de instalaciones eléctricas en mal estado y la exposición a gases y vapores, la magnitud del riesgo es alto junto con el temor; mientras que para el trabajo en alturas, por ejemplo, se encontró una alta magnitud del riesgo percibido y el resultado más bajo en el atributo de temor.

Lo anterior puede presentarse debido a que las actividades estudiadas por Slovic $(8,9,16)$, correspondían a aquellas situaciones cotidianas a las que se expone la población en general, mientras que las actividades objeto de este estudio,corresponden a aquellas específicas a la población trabajadora en el sector de la construcción, donde se pueden encontrar mayores beneficios para los expuestos, lo que 
implique que el temor se vea disminuido en muchas de las actividades evaluadas por este estudio.

Otro atributo que se menciona en los estudios realizados por Slovic(8)es el conocimiento por los expuestos, donde en la mayoría de los estudios realizados ha encontrado que se percibe mayor riesgo en aquellas actividades que se perciben como más conocidas. Sin embargo, en este estudio el atributo conocido por los expuestos se encontró para todas las actividades evaluadas un valor alto de conocimiento, y no así en la magnitud del riesgo percibido donde las actividades de exposición a ruido, exposición a polvo, desorden en la obra y manipulación de objetos y herramientas presentaron una magnitud de riesgo baja.

De acuerdo a los resultados obtenidos en este estudio, se evidencia que cuando se percibe que las consecuencias de alguna actividad son inmediatas los trabajadores manifiestan que la magnitud del riesgo es más alta, mientras que al percibir que las consecuencias son demoradas, la gravedad del daño se percibe como menor.

En este sentido los trabajadores estarían aludiendo a un sentimiento más de inmediatez de las consecuencias para determinar la gravedad del daño, que a un grado de temor o rechazo por aquellas actividades que se consideren como más dañinas para la salud y el bienestar, como lo que se encontró en los estudios tradicionales de percepción del riesgo(16-17).

Brun(18)un investigador que realizó estudios desde el paradigma psicométrico con riesgos de origen natural como enfermedades y desastres naturales, encontró que los atributos que se relacionaron con la magnitud de riesgo correspondían a novedad y efectos retardados, los mismos que se presentan es este estudio realizados con riesgos de origen laboral.

Otro de los resultados obtenidos por Brun(18) en sus estudios, es que las actividades con mayor magnitud del riesgo correspondieron a las actividades con mayor impacto individual que catastrófico. Mientras que en los resultados obtenidos por Slovic(8) las actividades con mayor magnitud del riesgo fueron también las de mayor impacto catastrófico.

Los resultados de este estudio indican que en algunas actividades con mayor magnitud de riesgo percibido también se perciben un alto potencial catastrófico como fue el caso de trabajar en espacios de baja iluminación, el uso de instalaciones eléctricas en mal estado y la exposición a gases y vapores. Pero para otras actividades cuando se manifestó mayor magnitud del riesgo se percibió bajo potencial catastrófico como en el caso del trabajo en alturas, el uso de herramientas cortantes y el no usar los elementos de protección personal.

El trabajo de investigación realizado por Puy(4), encontró que las actividades en las que se percibía mayor posibilidad de control, fueron por lo general, aquellas con una magnitud de riesgo más baja. En este estudio para el total de las actividades los trabajadores percibieron un alto posibilidad de control, pero algunas también se percibieron con una magnitud de riesgo alta.

En diversos estudios, tanto los realizados por Slovic(8), como el de Puy(4) se encontró que en general los sujetos de estudio tienden a valorar cada uno de los riesgos originados por las actividades como mejor conocido por los expertos que por las personas expuestas. Este tema también se encuentra en este estudio, donde aunque los resultados no presentan grandes diferencias en las medias de los atributos conocido por los expuestos y conocido por el experto, para todas las actividades la media más alta correspondió al ítem conocido por los expertos.

Un análisis que se realiza en los estudios a patir del paradigma psicométrico(4), consiste en la descripción de un perfil de los atributos percibidos para cada una de las actividades evaluadas. Lo anterior ha permitido comparar los resultados de los 
diferentes estudios identificando si el perfil de los atributos es similar en los diferentes estudios.

Sin embargo, debido a que las actividades elegidas para este estudio pertenecen exclusivamente al ámbito laboral del sector de la construcción y los estudios revisados solo han tenido en cuenta actividades cotidianas, no se llevaron a cabo comparaciones con los datos provenientes de las actividades estudiadas por otras investigadores.

En términos generales esta investigación ofrece una reflexión acerca de cuáles son los atributos que llevan a percibir un riesgo. En especial sugiere que el conocer los riesgos a los que las personas están expuestas, parece no tenerse en cuenta por los trabajadores a la hora de juzgar el hecho de exponerse o no exponerse.

Los trabajadores refieren que la posibilidad de prevenir los riesgos es alta. Frente a las cifras de accidentalidad en el sector, es imprescindible que los responsables formulen, realicen y evalúen acciones de intervención sustentadas en la percepción del riesgo, en las técnicas disponibles y en los criterios epidemiológicos.

De acuerdo a lo anterior se considera necesario realizar un tratamiento diferente a cada uno de los riesgos que se generan en la población trabajadora, y no solamente mencionar sus efectos y formas de prevención, pues el estudio indica que esto no ayuda a que el personal perciba el riesgo como algo que realmente le causa daño y deba sentir preocupación por exponerse a él.

Este estudio plantea la necesidad de estudiar los atributos de cada riesgo por separado e identificar la mejor manera de prevenirlos, encontrando que si el trabajador no percibe una magnitud y lo considera algo familiar del cual posee demasiado control de sus efectos, puede decidir arriesgarse mucho más, agregando a este aspecto el tema de los beneficios recibidos por su exposición como los mencionan algunos autores.

En este sentido es necesario para la prevención del riesgo, centrarse en cómo se percibe cada uno de los riesgos a los que son expuestos los trabajadores más que en el riesgo en sí y el daño que éste pueda causar, ya que por lo general no es el atributo que se evalúa la mayoría de las veces cuando el trabajador se expone.

Un aspecto al que se le podría dar mayor peso con el fin de realizar posteriores estudios de percepción del riesgo, corresponde al atributo de la inmediatez, ya que se evidenció que en general los trabajadores enunciaron mayor magnitud del riesgo en aquellos más inmediatos. Es decir en aquellos donde sus efectos se ven considerablemente másrápidos.

Si se revisa el tema de vulnerabilidad planteado por Morillejo(1), los trabajadores tomarán medidas de acuerdo a la vulnerabilidad que ellos perciban y en este caso el estudio indica que los trabajadores pueden no sentirse vulnerables, ya que aunque la magnitud del riesgo percibida es alta, perciben control sobre los riesgos, y un conocimiento del mismo que le permite enfrentarse a él. Así los sistemas de promoción de la salud deben estar enfocados a que el trabajador perciba su vulnerabilidad y realice una estimación para saber cómo actuar y donde detenerse.

Finalmente,al identificar los atributos subyacentes a la percepción del riesgo, se encontró que para cada uno de los riesgos entran en juego determinadas características y que es importante su reconocimiento para saber cómo actuar en la prevención de la accidentalidad. Si se tienen en cuenta estos atributos se podría identificar qué tan vulnerable se percibe la población con la que se pretende trabajar y de esta manera encaminar acciones al desarrollo de ciertas características que permitan una mayor prevención del riesgo. 


\section{Referencias}

1. Morillejo EA, Muñoz CP, Almería U De. La percepción del riesgo en la prevención de accidentes laborales. Apunt Psicol [Internet]. 2002;20(3):415-126. Available from: http://www.cop.es/delegaci/andocci/files/contenidos/ VOL20_3_6.pdf

2. Slovic P, Finucane ML, Peters E, MacGregor DG. Risk as analysis and risk as feelings. Risk Anal [Internet]. 2004;24(2). Available from: papers2://publication/uuid/ F4B3EA59-C2F7-4F62-9223-701C27F152C2

3. Sjöberg L. Factors in Risk Perception. Risk Anal ysis [Internet]. 2000;20(1-12). Available from: http:// www.readcube.com/articles/10.1111\%2 F02724332.00001?r3_referer=wol\&tracking_action=preview_ click\&show_checkout $=1$

4. Puy RA. Percepción social del riesgo: Dimensiones de evaluación y predicción. Universida. 2000. 408 p.

5. Wildavsky a., Dake K. heories of risk perception: Who fears what and why? Daedalus [Internet]. 1990;119(4):41-60. Available from: http://www.jstor.org.ezproxy.unal.edu.co/ stable/20025337? Search=yes\&resultItemClick=true\&searc hText="Theories of Risk Perception: Who Fears What and Why?" \&searchUri=/action/doBasicSearch?Query=\%22The ories+of+Risk+Perception $\% 3 \mathrm{~A}+$ Who $\%$

6. Slovic P. Perception of Risk Author ( $s$ ): Paul Slovic. Science (80- ) [Internet]. 1987;236(4799):280-5. Available from: http://www.jstor.org.ezproxy.unal.edu.co/stable/ pdf/1698637.pdf?acceptTC=true

7. Bickerstaff K. Risk perception research: socio-cultural perspectives on the public experience of air pollution. Environ Int [Internet]. 2004 Aug [cited 2015 Jun 25];30(6):82740. Available from: http://www.sciencedirect.com/science/ article/pii/S0160412003002472

8. Slovic P. The perception of risk. London: Ea. 2000. 473 p.

9. Slovic P, Fischhoff B, Lichtenstein S. Why study risk perception? [Internet]. Risk analysis. 1982. p. 8393. Available from: http://onlinelibrary.wiley.com/ doi/10.1111/j.1539-6924.1982.tb01369.x/full

10. Remoundou K, Brennan M, Sacchettini G, Panzone L, Butler-Ellis MC, Capri E, et al. Perceptions of pesticides exposure risks by operators, workers, residents and bystanders in Greece, Italy and the UK. Sci Total Environ [Internet].
2015 Feb 1 [cited 2015 Jun 25];505:1082-92. Available from: http://www.sciencedirect.com/science/article/pii/ S0048969714015605

11. Blanco A, Sánchez F, Carrera P CA y RD. Supuestos teóricos para un modelo psicosocial de las conductas de riesgo. In: Yubero, S. y Larrañaga E, editor. Sida: Una visión multidisciplinar. España: Universidad Castilla La Mancha; 2000. p. 41-62.

12. Elkind PD. Perceptions of risk, stressors, and locus of control influence intentions to practice safety behaviors in agriculture. J Agromedicine [Internet]. 2007;12(4):7-25. Available from: http://eds.b.ebscohost.com.ezproxy.unal.edu. $\mathrm{co} /$ ehost/pdfviewer/pdfviewer?sid=c4f223d3-f725-40d780e8-e628b5780b56\%40sessionmgr115\&vid=4\&hid $=108$

13. Arezes PM, Miguel AS. Hearing protection use in industry: The role of risk perception. Saf Sci [Internet]. 2005 Apr [cited 2015 Jun 25];43(4):253-67. Available from: http://www.sciencedirect.com/science/article/pii/ S0925753505000354

14. Gürcanlı GE, Baradan S, Uzun M. Risk perception of construction equipment operators on construction sites of Turkey. Int J Ind Ergon [Internet]. 2015 Jan [cited 2015 Feb 22];46:59-68. Available from: http://www.sciencedirect. com/science/article/pii/S0169814114001711

15. Canney P. Seguridad y salud en el trabajo de construcción: El caso Colombia [Internet]. 2000. Available from: http:// white.lim.ilo.org/spanish/260ameri/publ/docutrab/dt129/dt_129.pdf

16. Slovic P, Fischhoff B, Lichtenstein S. Characterising perceived risk. Perilous progress: Managing the Hazards of Technology. 1985. p. 91-125.

17. Fischhoff B, Watson SR, Hope C. Defining risk. Policy Sci [Internet]. 1984;17(2):123-39. Available from: http:// www.researchgate.net/publication/227182597_Defining_ risk

18. Brun Wibecke. Cognitive components in risk perception: Natural versus manmade risks. J Behav Decis Mak [Internet]. 1992;5(2):117-32. Available from: http:// onlinelibrary.wiley.com/doi/10.1002/bdm.3960050204/ citedby\#fn 1 